\title{
AVALIAÇÃO DO USO DE CARBONO ATIVADO COMO SUPORTE PARA CATALISADOR À BASE DE FERRO PARA SÍNTESE DE FISCHER-TROPSCH
}

\author{
M. G. A. da CRUZ ${ }^{1}$, F. A. N. FERNANDES ${ }^{2}$, M. BASTOS-NETO ${ }^{3}$ e A. C. OLIVEIRA ${ }^{4}$ \\ ${ }^{1,2,3}$ Universidade Federal do Ceará, Departamento de Engenharia Química \\ ${ }^{4}$ Universidade Federal do Ceará, Departamento de Química \\ E-mail para contato: gabrielycruz@yahoo.com.br
}

\begin{abstract}
RESUMO - Um carbono obtido a partir da carbonização de poliestireno reticulado e ativado por tratamento térmico com vapor foi utilizado como suporte para um catalisador de ferro para síntese de Fischer-Tropsch. O catalisador, com razão molar de 100Fe:163C, foi caracterizado por DRX, FRX, BET e XPS e submetido a testes catalíticos, utilizando diferentes condições de temperatura $(513,528$ e $543 \mathrm{~K})$ e pressão (20 e $25 \mathrm{~atm})$. As caracterizações evidenciaram a impregnação da fase metálica no suporte porém aquém do percentual de ferro calculado. As análises expuseram também a semi-cristalinidade do catalisador bem como a fase ativa presente, $\alpha-\mathrm{Fe}_{2} \mathrm{O}_{3}$. Parte dela está depositada na superfície do suporte, evidenciada pela diminuição nos valores de área específica, volume e diâmetro de poros obtidos através da análise das características texturais. Os testes catalíticos apresentaram boa seletividade em alta temperatura para hidrocarbonetos maiores que $\mathrm{C}_{5}$, porém houve metanação em baixa pressão e temperatura.
\end{abstract}

\section{INTRODUÇÃO}

A síntese de Fischer-Tropsch consiste na conversão do gás de síntese em hidrocarbonetos de alto peso molecular, utilizando-se catalisadores de $\mathrm{Fe}$ e Co a temperaturas entre 180 e $360{ }^{\circ} \mathrm{C}$ e pressões em torno de 45 atm, conforme as Equações 1 e 2, a seguir (Dry, 1990).

$$
\begin{aligned}
& \mathrm{nCO}+(2 \mathrm{n}+1) \mathrm{H}_{2} \rightarrow \mathrm{C}_{\mathrm{n}} \mathrm{H}_{(2 \mathrm{n}+2)}+\mathrm{nH}_{2} \mathrm{O} \\
& n \mathrm{CO}+2 n \mathrm{H}_{2} \rightarrow \mathrm{C}_{n} \mathrm{H}_{2 n}+n \mathrm{H}_{2} \mathrm{O}
\end{aligned}
$$

Os principais produtos são $\alpha$-olefinas e parafinas, principalmente de cadeia linear, mas há também produtos secundários oxigenados, tais como, alcoóis, cetonas e ácidos carboxílicos (Oliseev, 2009). Em elevadas temperaturas há favorecimento da formação de benzeno e seus homólogos. Essas reações ocorrem na superfície do catalisador de duas maneiras: por propagação de cadeia, via adição de um monômero a um intermediário previamente formado, e por terminação de cadeia.

A reação se dá pela polimerização de monômeros de metileno, como foi proposto originalmente 


\section{9 a 22 de outubro de 2014 \\ Florianópolis/SC}

por Fischer e Tropsch. Pode ocorrer também pela adsorção e dissociação de CO na superfície do catalisador seguida da adição do hidrogênio na superfície do átomo de carbono, levando à formação do monômero (Fontenelle; Fernandes, 2011).

Somente catalisadores de $\mathrm{Fe}$ e Co são utilizados comercialmente, dada a desvantagem apresentada pelo custo do Ru. Além disso, estudos revelaram uma atividade maior do Co com relação ao Fe (Li et al., 2002). Porém, neste, como em outros trabalhos, utiliza-se o Fe como fase ativa do catalisador.

$\mathrm{O} \mathrm{Fe}$ apresenta duas rotas de seletividade para a produção de hidrocarbonetos. Uma, denominada de alta temperatura, opera em aproximadamente $270{ }^{\circ} \mathrm{C}$, na qual há favorável produção de hidrocarbonetos olefínicos de baixo peso molecular mesmo na fase líquida. E a outra, de baixa temperatura, opera a $240{ }^{\circ} \mathrm{C}$. Nesta última há maior produção de hidrocarbonetos na fase líquida, sendo a principal fração de produto de parafina (Schulz e Cronjé, 1977). Além disso, essa rota também é bastante seletiva para o hidrocraqueamento na produção de diesel (Farias, 2007). Assim, essa reação pode ocorrer de duas maneiras: i) a baixas temperaturas, havendo o favorecimento da produção de graxas, que posteriormente são convertidas a naftas ou óleo diesel por hidroprocessamento (Espinoza et al., 1999); ii) a elevadas temperaturas, quando há maior conversão em gasolina e $\alpha$-olefinas (Steynberg, et al., 1999)

O objetivo desta pesquisa é estudar o eomportamente-desempenho de um catalisador de ferro suportado em carbono ativado das condiçães operacionais-na produção de hidrocarbonetos via síntese Fischer-Tropsch utilizando um catalisador de ferro suportado em carbono ativado. Utilizande pP्arâmetros como pressão e temperatura foram variados para avaliar esse $\underline{\text { o c comportamento desse }}$ catalisador.

\section{MATERIAL E MÉTODOS}

\subsection{Suporte}

A síntese dos suportes foi realizada em laboratório pela empresa alemã Blücher®. O suporte de carbono $\mathrm{CP} 1$, um carbono ativado esférico à base de polímero, foi obtido utilizando-se poliestireno reticulado como precursor, o qual foi carbonizado e ativado com vapor em um forno rotativo (Von Blücher, 2006).

A etapa de ativação com vapor permite que se determine o volume de poro através de uma oxidação controlada a $1173 \mathrm{~K}$. O aumento do tempo de ativação permite a oxidação do carbono com vapor e a consolidação do sistema de poros, o que influencia diretamente no volume total de poros do suporte que, segundo a regra de Gurvich, é um indicativo do grau de ativação (Rouquerol et al., 1999).

O volume de poros obtido para amostra CP1 foi de $1,2 \mathrm{~cm}^{3} \mathrm{~g}^{-1}$. A distribuição do tamanho de poros resultou em diâmetros na faixa de 0,7 a $3 \mathrm{~nm}$, porém uma pequena quantidade de poros encontra-se entre 10 e $20 \mathrm{~nm}$. 


\subsection{Síntese de Catalisadores}

O catalisador foi obtido pelo método de impregnação à umidade incipiente visando razão molar de 100Fe: 163C. Uma solução de nitrato de ferro foi adicionada lentamente ao suporte em um evaporador rotativo, a uma temperatura de $70{ }^{\circ} \mathrm{C}$ e vácuo de $500 \mathrm{mmHg}$, visando-tendo em vista a completa impregnação. Após esse processo, o catalisador seguiu para secagem em estufa a $60^{\circ} \mathrm{C}$ até que a solução atingisse completa secagem. Em seguida, o catalisador foi calcinado a temperatura de $500{ }^{\circ} \mathrm{C}$ por 4 horas em atmosfera ambiente (ar).

\subsection{Síntese de Fischer-Tropsch}

As reações de síntese de Fischer-Tropsch foram realizadas em um reator leito de lama (modelo PARR), o qual possui um sistema de agitação por acoplamento magnético que permite a recirculação da fase gás através da fase lama. Esse reator possui também chicanas para minimizar a formação de vórtice.

$\mathrm{O}$ reator foi alimentado com $2 \mathrm{~g}$ do catalisador de ferro suportado em carbono ativado a base de polímeros e $200 \mathrm{~mL}$ de esqualeno, um composto de hidrocarbonetos na faixa do $\mathrm{C}_{30}$ que constitui a fase líquida inerte. Monóxido de carbono e hidrogênio foram alimentados ao reator em fluxo segundo batelada alimentada através de controladores de fluxo mássico. A agitação do reator foi ajustada para $760 \mathrm{rpm}$, e as temperaturas e pressões variaram de acordo com os experimentos, como descrito na tabela 1.

A ativação do catalisador foi realizada in situ em um tempo de 4 horas de reação. Amostras da fase gasosa e da fase líquida foram analisadas via cromatografia gasosa em um cromatógrafo a gás (Thermos Ultra).

O planejamento experimental seguiu um planejamento fatorial quadrado $\left(2^{2}\right)$ com ponto central, no qual as temperaturas variaram entre 240 e $270{ }^{\circ} \mathrm{C}$, e a pressão, entre 20 e 25 atm, descritas na Tabela 1. Utilizou-se elevada pressão pelo fato de o catalisador de ferro ser bastante seletivo nessa condição para hidrocarbonetos de cadeia longa e por ser a condição próxima à utilizada industrialmente. A escolha das temperaturas deu-se pelo fato de serem relatadas na literatura como temperaturas ótimas, já que a $240^{\circ} \mathrm{C}$ há o favorecimento de produção de hidrocarbonetos pesados, tais como diesel e graxa, e a $270^{\circ} \mathrm{C}$ o mesmo ocorre com gasolina e querosene (Steynberg, 1999). 
Tabela 1 - Tabela de condições avaliadas segundo planejamento experimental fatorial quadrado com ponto central.

\begin{tabular}{c|cc}
\hline Corrida & Temperatura $\left({ }^{\circ} \mathbf{C}\right)$ & Pressão $(\mathbf{a t m})$ \\
\hline $\mathbf{1}$ & 240 & 20 \\
$\mathbf{2}$ & 270 & 20 \\
$\mathbf{3}$ & 255 & 25 \\
$\mathbf{4}$ & 240 & 25 \\
$\mathbf{5}$ & 270 & 25 \\
\hline
\end{tabular}

\section{RESULTADOS E DISCUSSÃO}

\subsection{Caracterização dos catalisadores}

A amostra do catalisador obtida (FeCP1) foi caracterizada via DRX, FEX, BET e XPS.

A análise de FRX evidenciou a considerável presença de Fe, com 71,43\%. O percentual restante deve-se à presença de carbono e oxigênio não detectado pela técnica de caracterização.

Após refinamento, o resultado da análise de DRX é mostrado na figura 01, bem como o resultado obtido para uma amostra de catalisador de Fe e sílica. Tais gráficos são obtidos a partir do programa X-pert Highscore Plus e refinados através do software DBWSTools, que utiliza o método de refinamento de Rietveld.

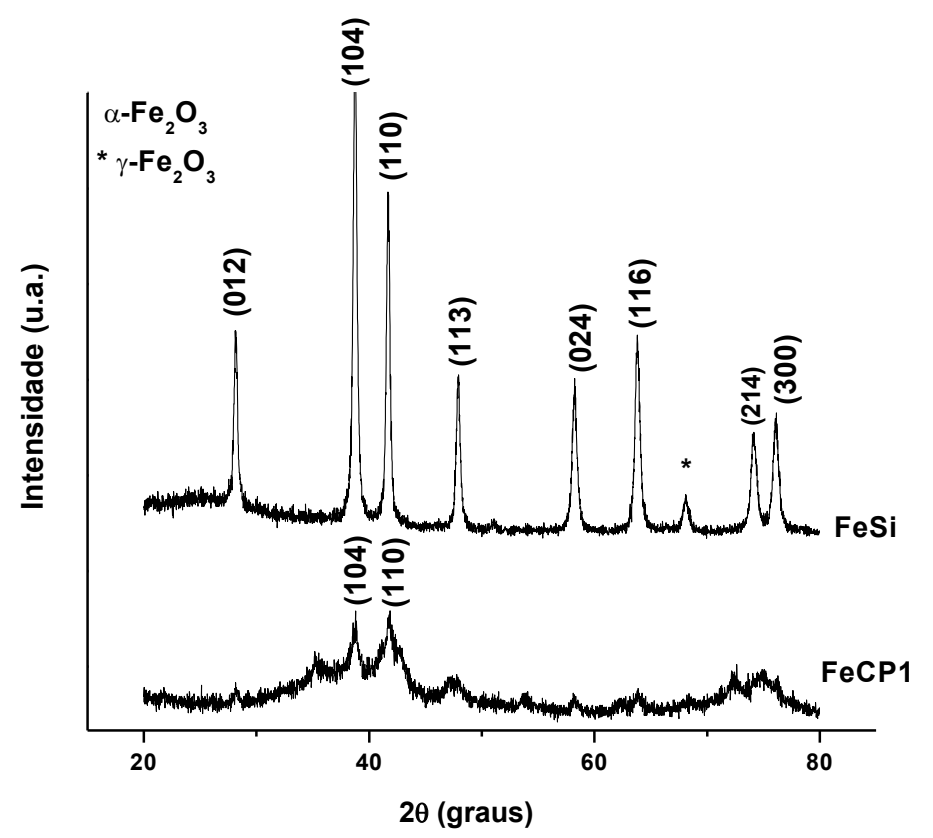

Figura 01 - Difratograma das amostras de catalisador de FeSi e FeCP1. 
A análise comparativa dos difratogramas obtidos evidencia o caráter semi-cristalino da amostra com carbono. Entretanto, apesar dessa característica, evidencia-se a presença do óxido de ferro III como fase ativa.

O percentual da fase ativa presente no catalisador pode ser determinado pela análise de XPS, bem como o estado dos elementos presentes na sua superfície. A tabela 03 apresentam os dados obtidos para carbono e oxigênio a partir dos gráficos da figura 02. Os grupos funcionais oxigenados são evidenciados tanto nos dados de $\mathrm{C} 1 \mathrm{~s}$ como nos de $\mathrm{O} 1 \mathrm{~s}$. Observa-se a presença de óxido de ferro na amostra através dos dados de oxigênio $\mathrm{O}$ 1s, na tabela 03, e de percentual atômico de ferro na tabela 04, pois este apresenta-se aqui associado em forma de óxido.

Os picos descritos na figura 02 (c) somados à presença de um pico satélite próximo a $719 \mathrm{eV}$ confirmam que a espécie de ferro predominante na superfície é o $\mathrm{Fe}^{3+}$, como mostrado por Rey (2009) na caracterização de um catalisador suportado em carbono ativado advindo de fonte natural.

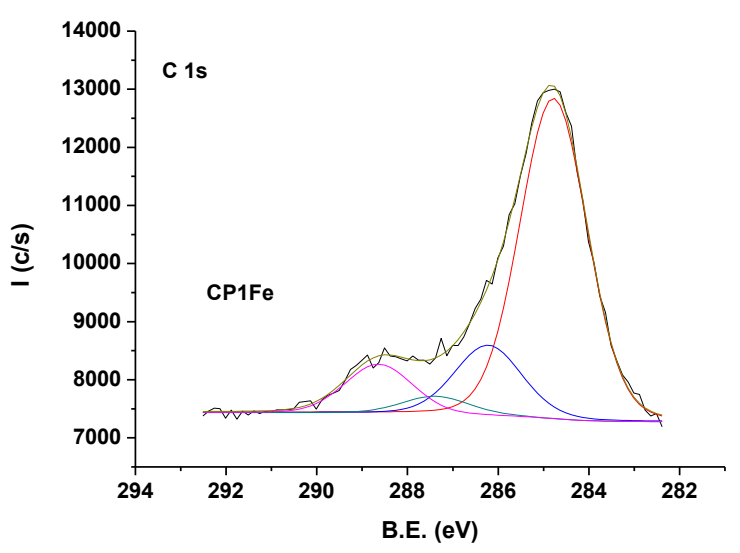

(a)

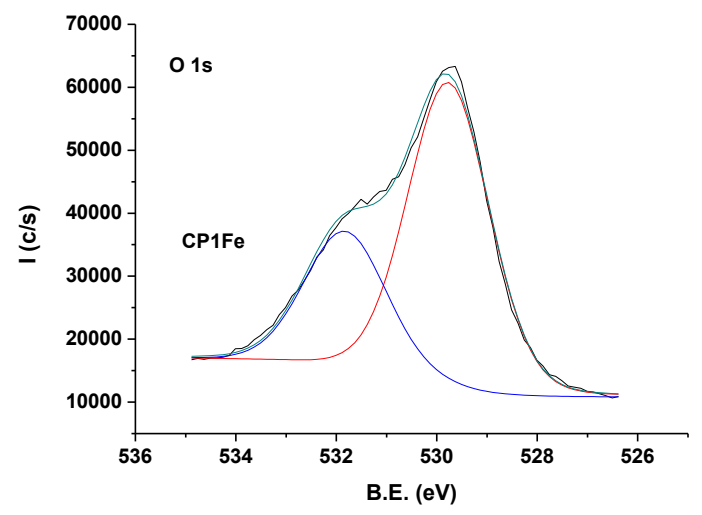

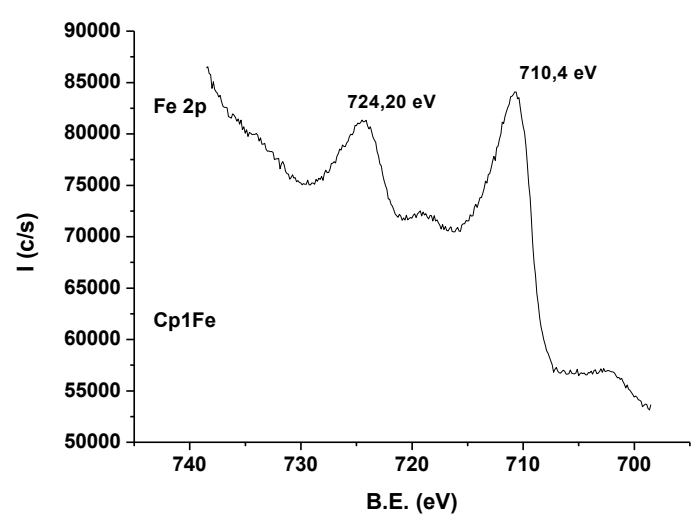

(c) (b)

Figura 02 - Espectros de XPS para amostra do catalisador FeCP1 para (a) C 1s;

(b) $\mathrm{O} 1 \mathrm{~s}$ e (c) Fe 2p. 
Tabela 03 - Resultado de deconvolução dos dados de XPS para as amostras obtidas

\begin{tabular}{|c|c|c|c|c|}
\hline \multirow{2}{*}{ Região } & \multirow{2}{*}{ Pico } & \multicolumn{3}{|c|}{ Catalisador FeCP1 } \\
\hline & & Posição (eV) & Área $(\%)$ & Estado do elemento \\
\hline \multirow{5}{*}{$\mathrm{C} 1 \mathrm{~s}$} & $\mathrm{C} 1$ & 284,7 & 74,07 & Grafite \\
\hline & $\mathrm{C} 2$ & 286,3 & 15,95 & $-\mathrm{C}-\mathrm{OH} / \mathrm{-C}-\mathrm{O}$ \\
\hline & $\mathrm{C} 3$ & 288,4 & 9,98 & $-\mathrm{C}=\mathrm{O}-\mathrm{I}-\mathrm{O}-\mathrm{C}-\mathrm{O}-$ \\
\hline & $\mathrm{C} 4$ & 288,9 & 3,01 & $\mathrm{O}-\mathrm{C}=\mathrm{O}$ \\
\hline & $\mathrm{C} 5$ & 290,5 & 1,97 & $\pi-\pi *$ \\
\hline \multirow{2}{*}{$\mathrm{O} 1 \mathrm{~s}$} & O1 & 529,8 & 84,79 & $\mathrm{Fe}_{2} \mathrm{O}_{3}$ \\
\hline & $\mathrm{O} 2$ & 531,6 & 15,21 & $\mathrm{FeOOH}\left(\mathrm{Fe}_{2} \mathrm{O}_{3} \cdot \mathrm{H}_{2} \mathrm{O}\right)$ \\
\hline
\end{tabular}

Tabela 04 - Dados de concentração atômica em percentual obtidos pela técnica de XPS dos elementos presentes nas amostras.

\begin{tabular}{cccc}
\hline & C 1s & O 1s & Fe 2p \\
\hline CP1 & 91,68 & 8,32 & - \\
FeCP1 & 18,28 & 66,76 & 14,31 \\
\hline
\end{tabular}

A análise de BET do carbono ativado antes da impregnação com Fe, mostrada na tabela 05, seguindo as recomendações da IUPAC, mostra que o carbono é predominantemente formado por mesoporos $(20-500 \AA$ ) ), porém com presença de microporos, evidenciados após impregnação. A diminuição da área superficial -expõe a deposição da fase ativa na superfície do suporte, o que pode propiciar a formação de aglomerados. Tal fato pode ser confirmado por a amostra ter se tornado microporosa e pela considerável diminuição do volume de poros.

Tabela 05 - Resultado da análise BET

\begin{tabular}{c|ccc}
\hline & $\begin{array}{c}\text { Área } \\
\text { superficial }\end{array}$ & $\begin{array}{c}\text { Diâmetro } \\
\text { do poro }\end{array}$ & $\begin{array}{c}\text { Volume de } \\
\text { poro }\end{array}$ \\
\hline Suporte de carbono ativado & $2070 \mathrm{~m}^{2} / \mathrm{g}$ & $25,89 \AA$ & $1,28 \mathrm{~cm}^{3} \mathrm{~g}^{-1}$ \\
Suporte impregnado com Fe (Catalisador) & $172,8 \mathrm{~m}^{2} / \mathrm{g}$ & $11,69 \AA$ & $0,23 \mathrm{~cm}^{3} \mathrm{~g}^{-1}$ \\
\hline
\end{tabular}

\subsection{Síntese de Fischer-Tropsch}

Após o tempo de reação de 4 horas-e utilizando-se o catalisador de carbono polimérico, os produtos foram analisados via cromatografia gasosa, com cujos resultados pôde-se traçar o perfil de hidrocarbonetos produzidos em cada corrida. Os resultados dos perfis de hidrocarbonetos gerados via síntese Fischer-Tropsch são apresentados na tabela 06, a seguir. 
Tabela 06 - Perfil de concentração de hidrocarbonetos dos produtos obtidos.

\begin{tabular}{cccccc}
\hline Temperatura $\left({ }^{\circ} \boldsymbol{C}\right)$ & Pressão $(\boldsymbol{a t m})$ & H2:CO & $\boldsymbol{C 1 - C 4}$ & $\boldsymbol{C 5 - C \boldsymbol { 9 }}$ & $\boldsymbol{C 1 0 +}$ \\
\hline 240 & 20 & $1: 1$ & 95,65 & 1,49 & 2,87 \\
240 & 30 & $1: 1$ & 37,44 & 44,61 & 17,94 \\
255 & 25 & $1: 1$ & 59,42 & 16,08 & 24,50 \\
270 & 25 & $1: 1$ & 55,62 & 5,67 & 38,71 \\
270 & 30 & $1: 1$ & 73,74 & 8,27 & 26,96 \\
\hline
\end{tabular}

O perfil descreve uma reação que favoreceu a formação de hidrocarbonetos leves. Tal fato pode ser justificado pelos baixos valores de diâmetro e volume de poros apresentados pelo catalisador, por limitar a mobilidade de uma cadeia maior de carbonos nos poros do catalisador. Entretanto, a presença da hematita $\left(\mathrm{Fe}_{2} \underline{\mathrm{O}}_{3}\right)$ como fase ativa, evidenciada tanto na análise de DRX quanto em XPS, catalisa a reação para formação de hidrocarbonetos de alto peso molecular $\left(\mathrm{C}_{\underline{5+}}\right)$.

Analisando os perfis somente a partir de $\mathrm{C}_{5+2}$ vê-se uma preferência pela formação de cadeias com mais de 10 carbonos, com exceção da condição de $240{ }^{\circ} \mathrm{C}$ e $30 \mathrm{~atm}$. É possível que a temperatura correta para a formação de hidrocarbonetos entre $\underline{C}_{5}$ e $\underline{C}_{9}$ a elevada pressão e a presença da hematita, apesar do pequeno tamanho de poros, tenham levado à formação dessas cadeias de carbono. Isso ocorre porque a pressão pode manter a cadeia em formação dentro do poro até onde seu tamanho possa permitir. $\Theta$ catalisador FeCP1 apresentou maior seletividade para hidrocarbonetos com mais de 10 carbonos ao-comparar com os percentuais obtidos entre hidrocarbonetos de alto peso molecular (C5t). Tal fato pode ser associado às caracterísiticas apresentada pelo catalisador na análise de fisissorção de $\mathrm{N}_{z}$. Apesar de apresentar um menor percentual de fase ativa na sua superfície, o fato de o seu diâmetro de poro-e área superficial serem maiores permite a mobilidade de uma cadeia maior de carbono nos poros do catalisador.

Catalisadores de ś́lica foram testados variando a carga de metal, dentre as quais $50 \% \mathrm{em}$ massa de ferro, similar à carga utilizada na pesquisa. _Pirola (2009) estudou catalisadores de Ferro suportados em sílica usando temperatura de $\mathrm{A}$ seletividade desse catalisador foi avaliada eөm as seguintes condições de reação: $523 \mathrm{~K}$; razão molar $\mathrm{H}_{2}: \mathrm{CO}=2$ e pressão de $22_{2}-0 \mathrm{Mpa}$. O catalisador com teor de metal semelhante obteve para o metano uma seletividade de $6 \% ; 20 \%$ para hidrocarbonetos menores que $\mathrm{C}_{7}$, e $52 \%$ para compostos maiores que $\mathrm{C}_{7}$. Apesar de diferir quanto à razão molar do gás de síntese, o percentual obtido para compostos $\mathrm{C}_{7+}$ é similar ao obtido para o catalisador $\mathrm{FeCP} \underline{1} z$ em condições similares de temperatura e pressão, sendo de 543 $\mathrm{K}$, 20 bar $25 \mathrm{~atm}$ e $\mathrm{H}_{2}: \mathrm{CO}=1$ com $65,32 \%$ com aproximadamente $45 \%$ de concentração para $\mathrm{o}$ $\mathrm{C}_{5+}$. 


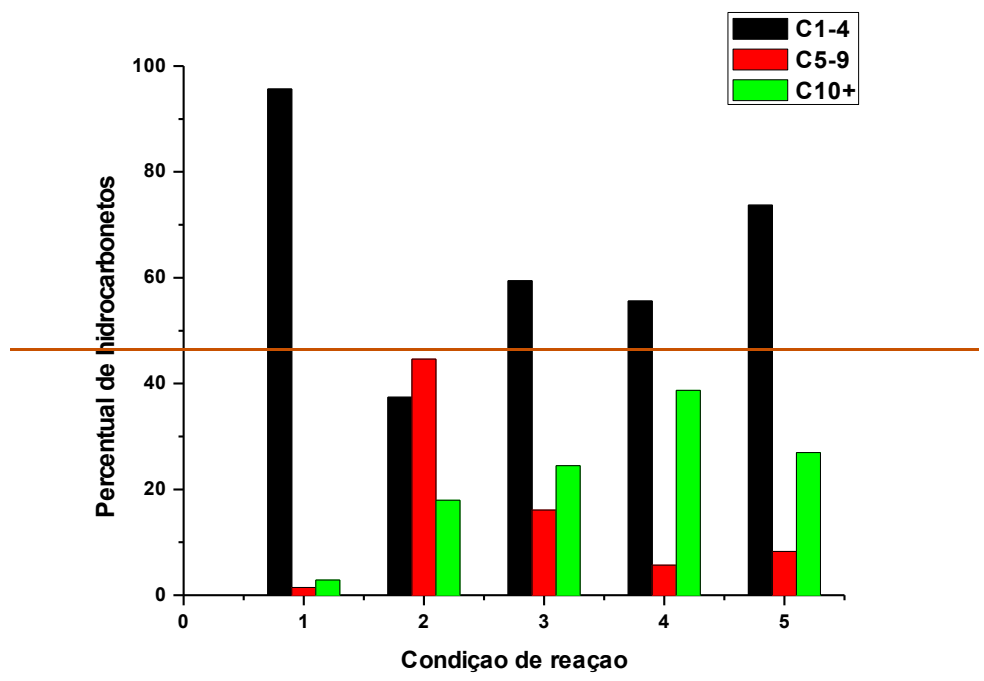

Figura 03 Perfil de concentração de hidrocarbonetos obtido para o catalisador FeCP1 em diferentes condiçẽes de temperatura e razão molar $\mathrm{H}_{2}: \mathrm{CO}=1$.

\section{CONCLUSÃO}

A utilização do carbono ativado como suporte para catalisador de ferro para síntese de FischerTropsch mostrou-se viável. As caracterizações do catalisador evidenciaram presença da fase ativa na superfície do suporte e a microporosidade do catalisador. Essa característica pode justificar o favorecimento na produção de hidrocarbonetos leves, chegando, em uma das condições avaliadas, a aproximar-se de uma metanação. Porém houve produção de hidrocarbonetos pesados, $\mathrm{C}_{5+-9} \mathrm{e}$, dentre esses, tem-se maior produção de hidrocarbonetos pesados, $\mathrm{C}_{10+}$, com o aumento da temperatura.

\section{REFERÊNCIAS BIBLIOGRÁFICAS}

DAVIS, B. H.; Fischer-Tropsch synthesis: relationship between iron catalyst composition and process variables. Catalysis Today, 2003, v. 84, n. 1-2, p. 83-98.

DRY, M. E. The fischer-tropsch process - commercial aspects. Catalysis Today, v. 6, n. 3, p. 183-206, 1990.

ESPINOZA, R. L.; STEYNBERG, A. P.; JAGER, B.; VOSLOO, A. C.; Low temperature FischerTropsch synthesis from a Sasol perspective. Applied Catalysis A: General, 1999, v. 186, n. 1-2, p. 13 26.

FARIAS, F. E. M., Produção de hidrocarbonetos através da Síntese de Fischer-Tropsch utilizando catalisadores de Fe/K. Dissertação de Mestrado em Engenharia Química, Universidade Federal do Ceará, 2007.

FONTENELLE JR, A. B.; FERNANDES, F. A. N.; Comprehensive polymerization model for 
Fischer-Tropsch Synthesis. Chemical Engineering Technology, 2011 v. 34, n. 6, p. 963-971.

LI, S.; DING, W.; MEITZNER, G. D.; IGLESIA, E.; Spectroscopic and Transient Kinetic Studies of Site Requirements in Iron-Catalyzed Fischer-Tropsch Synthesis. The Journal of Physical Chemistry B, 2002, v. 106, n. 1, p. 85-91.

OLISEEV, O. L.; Gas to Liquid Technologies. Russian Journal of General Chemistry, 2009, Vol. 79, No. 11, pp. 2509-2519.

PIROLA, C. E. et al. Fischer Tropsch and Water Gas Shift chemical regimes on supported iron-based catalysts at high metal loading. Catalysis Communications, v. 10, n. 6, p. 823-827, 2009.

REY, A. et al. Catalytic wet peroxide oxidation of phenol over Fe/AC catalysts: Influence of iron precursor and activated carbon surface. Applied Catalysis B: Environmental, v. 86, n. 1-2, p. 69-77, 2009.

ROUQUEROL, F. et al. Adsorption by Powders \& Porous Solids. 2. ed. San Diego, CA: Academic Press, 1999.

SCHULZ, H.; CRONJÉ, J. H.; Fischer-Tropsch Synthesis. Ullmanns Enzyklopadie der Technischen Chemie, 1977, v.14. 4th ed. Weinheim: Verlag Chemie, p. 329.

STEYNBERG, A. P.; ESPINOZA, R. L.; JAGER, B.; VOSLOO, A. C.; High temperature FischerTropsch synthesis in commercial pratice. Applied Catalysis A: General, 1999, v. 186, n. 1-2, p. 41-54.

UNMUTH, E. E.; SCHWARTZ, L. H.; BUTT, J. B.; Iron alloy Fischer-Tropsch catalysts. I. Oxidation-reduction studies of the FeNi system. Journal of Catalysis, 1980, 61, pp. 242-255.

VON BLÜCHER, H.; BÖHRINGER, B. DR.; GIEBELHAUSEN, J. M. High Performance adsorbents based on active carbon with high micro-porosity. EP1918022 A1. Alemanha, 07 maio, 2007. 